PROCEEDINGS OF THE

AMERICAN MATHEMATICAL SOCIETY

Volume 139, Number 7, July 2011, Pages 2415-2421

S 0002-9939(2010)10686-X

Article electronically published on December 9, 2010

\title{
CANONICAL FILTRATIONS OF GORENSTEIN INJECTIVE MODULES
}

\author{
EDGAR E. ENOCHS AND ZHAOYONG HUANG
}

(Communicated by Bernd Ulrich)

\begin{abstract}
The principle "Every result in classical homological algebra should have a counterpart in Gorenstein homological algebra" was given by Henrik Holm. There is a remarkable body of evidence supporting this claim. Perhaps one of the most glaring exceptions is provided by the fact that tensor products of Gorenstein projective modules need not be Gorenstein projective, even over Gorenstein rings. So perhaps it is surprising that tensor products of Gorenstein injective modules over Gorenstein rings of finite Krull dimension are Gorenstein injective.

Our main result is in support of the principle. Over commutative, noetherian rings injective modules have direct sum decompositions into indecomposable modules. We will show that Gorenstein injective modules over Gorenstein rings of finite Krull dimension have filtrations analogous to those provided by these decompositions. This result will then provide us with the tools to prove that all tensor products of Gorenstein injective modules over these rings are Gorenstein injective.
\end{abstract}

\section{INTRODUCTION}

Throughout this paper $R$ will denote a commutative and noetherian ring and $\operatorname{Spec}(R)$ will denote the set of its prime ideals. The term module will then mean an $R$-module. An injective envelope of the module $M$ will be denoted by $E(M)$ and

$$
0 \rightarrow M \rightarrow E^{0}(M) \rightarrow \cdots \rightarrow E^{n}(M) \rightarrow \cdots
$$

will denote a minimal injective resolution of $M$.

We will now give several definitions and results. For ease in referring to these later in the paper they will be numbered.

(1) Every injective module is uniquely up to isomorphism the direct sum of modules, each of which is isomorphic to $E(R / P)$ for some $P \in \operatorname{Spec}(R) 6$, Theorem 2.5 and Proposition 3.1].

(2) We say $R$ is a Gorenstein ring if $i n j, \operatorname{dim}_{R_{P}} R_{P}<\infty$ for each $P \in \operatorname{Spec}(R)$. If in fact $i n j . \operatorname{dim}_{R} R<\infty$, then $R$ is Gorenstein and the Krull dimension of $R$ equals inj.dim $R$ [1, Corollary 3.4].

(3) In inj.dim $_{R} R<\infty$ (and so $R$ is Gorenstein) and if $0 \rightarrow R \rightarrow E^{0}(R) \rightarrow$ $\cdots E^{n}(R) \rightarrow 0$ is a minimal injective resolution of $R$ as a module, then for

Received by the editors August 7, 2009 and, in revised form, July 5, 2010

2010 Mathematics Subject Classification. Primary 13D07, 16E30.

Key words and phrases. Gorenstein injective modules, torsion products, filtrations.

(C) 2010 American Mathematical Society Reverts to public domain 28 years from publication 
$0 \leq k \leq n, E^{k}(R)=\bigoplus_{h t(P)=k} E(R / P)$, where these $P$ are in $\operatorname{Spec}(R)$ [1, Proposition 3.6]. Furthermore when $h t(P)=k$, we have $f \operatorname{ld} \operatorname{dim} E(R / P)=$ $k$ [7, Theorem 5.1.2].

(4) If $R$ is Gorenstein and $E, E^{\prime}$ are injective modules, then for any $k \geq 0$ the module $\operatorname{Tor}_{k}\left(E, E^{\prime}\right)$ is injective. More precisely, if $P, Q \in \operatorname{Spec}(R)$, then $\operatorname{Tor}_{k}(E(R / P), E(R / Q))=0$ unless both $P=Q$ and $k=h t(P)$. Also in this case we have that $\operatorname{Tor}_{k}(E(R / P), E(R / P)) \cong E(R / P)$ [5, Lemma 2.1 and Theorem 4.1]. So using (1) we see that $\operatorname{Tor}_{k}(E(R / P), E)=0$ when $E$ is injective and $k \neq h t(P)$.

(5) If $P \in \operatorname{Spec}(R)$ a module $S$ will be said to have property $t(P)$ if for each $r \in R-P$ we have that $S \stackrel{r}{\rightarrow} S$ is an isomorphism and if for each $x \in S$ we have that $P^{m} x=0$ for some $m \geq 1$. If $S$ has property $t(P)$ and property $t(Q)$ with $P \neq Q$, then it is easy to see that $S=0$. If $S$ has property $t(P)$ and if $N$ is any module, then $\operatorname{Tor}_{k}(S, N)$ has property $t(P)$ for all $k \geq 0$. This can be seen by using a projective resolution of $N$ to compute this Tor. Consequently, if $S$ has property $t(P)$ and $T$ has property $t(Q)$ where $P \neq Q$, we get $\operatorname{Tor}_{k}(S, T)=0$ for all $k \geq 0$. For any $P \in \operatorname{Spec}(R)$ the module $E(R / P)$ has property $t(P)$ [6, Lemma 3.2].

(6) We now argue that if $S$ has property $t(P)$, then so does $E(S)$. By (1) above $E(S)$ is a direct sum of copies of $E(R / Q)$ for various $Q \in \operatorname{Spec}(R)$. If $r \in R-P$, then since $S \stackrel{r}{\rightarrow} S$ is an isomorphism, then so is $E(S) \stackrel{r}{\rightarrow} E(S)$. Now assume that $E(R / Q)$ is a summand of $E(S)$. Then for $r \in R-P$ we have that $E(R / Q) \stackrel{r}{\rightarrow} E(R / Q)$ is an isomorphism. Hence $r \in R-Q$. So we get $Q \subset P$. We want to argue that $Q=P$. If not, let $r \in P-Q$. The extension $S \rightarrow E(S)$ is essential, so the module $S^{\prime}=E(R / Q) \cap S$ is nonzero. Let $x \in S^{\prime}, x \neq 0$. Then since $x \in S$ and since $S$ has property $t(P)$, we get $P^{m} x=0$ for some $m \geq 1$. So $r^{m} x=0$. But $E(R / Q)$ has property $t(Q)$ and $r \in R-Q$. Hence $E(R / Q) \stackrel{r}{\rightarrow} E(R / Q)$ is an isomorphism. But then since $S^{\prime} \subset E(R / Q)$ we get that $S^{\prime} \stackrel{r}{\rightarrow} S^{\prime}$ is an injection. But this is not possible if $r^{m} x=0$, where $x \in S^{\prime}$ and $x \neq 0$. So we get $Q=P$.

So $E(S)$ is a direct sum of copies of $E(R / P)$, and so by (5) we see that $E(S)$ has property $t(P)$. But then the quotient module $E(S) / S$ will have property $t(P)$. So continuing we see that all the terms $E^{i}(S)(i \geq 1)$ in a minimal injective resolution of $S$ have property $t(P)$.

(7) If $S$ has property $t(P)$ and $T$ has property $t(Q)$ and if $P \not \subset Q$, then $\operatorname{Hom}(S, T)=0$. If $r \in P-Q$ and if $f(x)=y$ for some $f \in \operatorname{Hom}(S, T)$, we have $r^{n} x=0$ for some $n \geq 1$, and so $r^{n} y=0$. But since $r \notin Q$ this is possible only if $y=0$. So we get $f=0$.

(8) We recall that a module $G$ is said to be Gorenstein injective if and only if there is an exact sequence

$$
\cdots \rightarrow E_{2} \rightarrow E_{1} \rightarrow E_{0} \rightarrow E^{0} \rightarrow E^{1} \rightarrow E^{2} \rightarrow \cdots
$$

of injective modules with $G=\operatorname{Ker}\left(E^{0} \rightarrow E^{1}\right)$ and such that $\operatorname{Hom}(E,-)$ leaves the sequence exact whenever $E$ is an injective module. For the rest of (8) we assume that $R$ is a Gorenstein ring of finite Krull dimension $n$. If $n \geq 1$, a module $G$ is Gorenstein injective if and only if there is an exact sequence

$$
E_{n-1} \rightarrow \cdots \rightarrow E_{1} \rightarrow E_{0} \rightarrow G \rightarrow 0
$$


with $E_{n-1}, \ldots, E_{0}$ injective modules. This result gives that the class of Gorenstein injective modules over such $R$ is closed under arbitrary direct sums. Also if $n=0$, then every module $G$ is Gorenstein injective (see [4, Theorem 4.2] for both these claims). As a consequence we get that if $P$ is a minimal prime ideal of $R$ and if $G$ is an $R_{P}$-module, then $G$ is a Gorenstein injective $R$-module. This follows from the observation that $R_{P}$ is a flat $R$ module, so any injective $R_{p}$ module is also an injective $R$-module. Hence an exact sequence $\cdots E_{2} \rightarrow E_{1} \rightarrow E_{0} \rightarrow G \rightarrow 0$ of $R_{P}$-modules with the $E_{k}$ injective $R_{P}$-modules gives us an exact sequence of $R$-modules with the $E_{k}$ injective $R$-modules.

We need a slightly stronger version of the result above. So again we suppose $R$ is Gorenstein and of Krull dimension $n$ but with $n \geq 1$. We claim that if $G$ is such that there is an exact sequence

$$
G_{n-1} \rightarrow \cdots \rightarrow G_{0} \rightarrow G \rightarrow 0
$$

with $G_{n-1}, \ldots, G_{0}$ all Gorenstein injective, then $G$ is Gorenstein injective. By [6. Proposition 1.11], $G$ is Gorenstein injective if and only if $\operatorname{Ext}^{1}(L, G)=0$ whenever proj.dimL $<\infty$. By [4, Corollary 4.4], we have that proj.dim $L<\infty$ implies proj.dim $L \leq n$. So now using dimension shifting and these results we get that $G$ is Gorenstein injective.

(9) If $G$ is Gorenstein injective over any Gorenstein $R$ and $r \in R$ is $R$-regular, then $\operatorname{proj} \operatorname{dim} R /(r)=1$. So $\operatorname{Ext}^{1}(R /(r), G)=0$ by (8). This gives that $\operatorname{Hom}(R, G) \stackrel{r}{\rightarrow} \operatorname{Hom}(R, G) \rightarrow 0$ is exact. This means that $G \stackrel{r}{\rightarrow} G$ is surjective. So for every $x \in G$ there is a $y \in G$ with $r y=x$. Consequently we get that $G \otimes T=0$ if $T$ has property $t(P)$ and if $r \in P$. Also, if $x \in G$ and $y \in T$ and $n \geq 1$ we have that $x=r^{n} \bar{x}$ for some $\bar{x} \in G$. So $x \otimes y=r^{n} \bar{x} \otimes y=\bar{x} \otimes r^{n} y$. But if $n$ is sufficiently large we have $r^{n} y=0$. Hence $x \otimes y=0$.

Now if $P \in X$ and if $h t(P) \geq 1$, then since $R$ is Gorenstein (and so Cohen-Macaulay) there is an $R$-regular $r \in P$. Consequently we get that $G \otimes T=0$ whenever $G$ is Gorenstein injective and when $T$ has property $t(P)$ with $h t(P) \geq 1$.

\section{TORSION PRODUCTS OF INJECTIVE and Gorenstein injeCtive Modules}

In this section $R$ will be a Gorenstein ring of finite Krull dimension $n$. We let $X=\operatorname{Spec}(R)$. When we refer to $(1),(2), \ldots,(9)$ we mean the corresponding result in the preceding section.

Lemma 2.1. If $P \in X$ and $h t(P) \geq 1$, then for any Gorenstein injective module $G$ we have $E(R / P) \otimes G=0$.

Proof. By (5) we know that $E(R / P)$ has property $t(P)$. So this result is a special case of (9).

Proposition 2.2. If $G$ is Gorenstein injective and $P \in X$, then $\operatorname{Tor}_{k}(E(R / P), G)$ $=0$ if $h t(P) \neq k$.

Proof. By (3) we know that fl.dimE $(R / P)=h t(P)$, so $\operatorname{Tor}_{k}(E(R / P),-)=0$ if $k>h t(P)$. Therefore, we only need prove that $\operatorname{Tor}_{k}(E(R / P), G)=0$ when $G$ is Gorenstein injective and $k<h t(P)$. We prove this by induction on $k$. If $k=0$, then 
$\operatorname{Tor}_{k}(E(R / P), G)=E(R / P) \otimes G=0$ if $h t(P) \geq 1$ and $G$ is Gorenstein injective by Lemma 2.1 .

So now we make an induction hypothesis and let $h t(P)>k$ and let $G$ be Gorenstein injective. We have an exact sequence $0 \rightarrow H \rightarrow E \rightarrow G \rightarrow 0$ with $E$ injective and $H$ Gorenstein injective. We have the exact sequence $\operatorname{Tor}_{k}(E(R / P), E) \rightarrow$ $\operatorname{Tor}_{k}(E(R / P), G) \rightarrow \operatorname{Tor}_{k-1}(E(R / P), H)$. By the induction hypothesis and the fact that $h t(P)>k>k-1$, we have that $\operatorname{Tor}_{k-1}(E(R / P), H)=0$. But $\operatorname{Tor}_{k}(E(R / P), E)=0$ by (4), and so $\operatorname{Tor}_{k}(E(R / P), G)=0$.

Corollary 2.3. If $0 \rightarrow G^{\prime} \rightarrow G \rightarrow G^{\prime \prime} \rightarrow 0$ is an exact sequence of Gorenstein injective modules and if $E$ is an injective module, then for any $k \geq 0$ the sequence $0 \rightarrow \operatorname{Tor}_{k}\left(E, G^{\prime}\right) \rightarrow \operatorname{Tor}_{k}(E, G) \rightarrow \operatorname{Tor}_{k}\left(E, G^{\prime \prime}\right) \rightarrow 0$ is exact.

Proof. By (1) $E$ is a direct sum of submodules isomorphic to $E(R / P)$ with $P \in X$; it suffices to prove the claim when $E=E(R / P)$ for any $P$. In this case the claim follows by considering the long exact sequence of $\operatorname{Tor}(E(R / P),-)$ associated with $0 \rightarrow G^{\prime} \rightarrow G \rightarrow G^{\prime \prime} \rightarrow 0$ and Proposition 2.2.

Proposition 2.4. If $G$ is Gorenstein injective and $E$ is injective, then for any $k \geq 0$ the module $\operatorname{Tor}_{k}(E, G)$ is a Gorenstein injective module.

Proof. By (8) we have an exact sequence $\cdots \rightarrow E_{2} \rightarrow E_{1} \rightarrow E_{0} \rightarrow G \rightarrow 0$ with all the $E_{i}$ injective modules, where the kernels of $E_{0} \rightarrow G, E_{1} \rightarrow E_{0}, \ldots$ are Gorenstein injective. Therefore, we can split the exact sequence into short exact sequences $0 \rightarrow G_{1} \rightarrow E_{0} \rightarrow G \rightarrow 0,0 \rightarrow G_{2} \rightarrow E_{1} \rightarrow G_{1} \rightarrow 0, \ldots$ with each $G_{k}$ and $G$ Gorenstein injective. We then apply Corollary 2.3 and splice the resulting short exact sequences together to get the exact sequence $\cdots \rightarrow \operatorname{Tor}_{k}\left(E, E_{1}\right) \rightarrow$ $\operatorname{Tor}_{k}\left(E, E_{0}\right) \rightarrow \operatorname{Tor}_{k}(E, G) \rightarrow 0$. Since each $\operatorname{Tor}_{k}\left(E, E_{n}\right)$ is injective we get that $\operatorname{Tor}_{k}(E, G)$ is Gorenstein injective by the Proposition (8).

\section{Filtrations of Gorenstein injective modules}

We again let $R$ be a Gorenstein ring of finite Krull dimension $n$ and let $X=$ $\operatorname{Spec}(R)$ and let $X_{k} \subset X$ for $k \geq 0$ consist of the $P \in X$ such that $h t(P)=k$. In this section we will also appeal to the results $(1), \ldots,(9)$ of the first section.

The main contribution of this paper is the following result.

Theorem 3.1. If $G$ is a Gorenstein injective module, then $G$ has a filtration $0=G_{n+1} \subset G_{n} \subset \cdots \subset G_{2} \subset G_{1} \subset G_{0}=G$ where each $G_{k} / G_{k+1}(0 \leq k \leq n)$ is Gorenstein injective and has a direct sum decomposition indexed by $P \in X_{k}$ such that the summand, say $S$, corresponding to $P$ has the property $t(P)($ see (5)). Furthermore, such filtrations and direct sum decompositions are unique and functorial in $G$.

Proof. We first comment that "functorial in $G$ " means that if $H$ is another Gorenstein injective module with such a filtration $0=H_{n+1} \subset H_{n} \subset \cdots \subset H_{1} \subset H_{0}=H$ where $T$ is the summand of $H_{k} / H_{k+1}$ corresponding to $P \in X_{k}$ and if $f: G \rightarrow H$ is linear, then $f\left(G_{k}\right) \subset H_{k}$ for each $k$ and the induced map $G_{k} / G_{k+1} \rightarrow H_{k} / H_{k+1}$ maps $S$ (as in the theorem) into $T$. 
Now let $0 \rightarrow R \rightarrow E^{0}(R) \rightarrow \cdots \rightarrow E^{n}(R) \rightarrow 0$ be the minimal injective resolution of $R$ and let $\cdots \rightarrow P_{2} \rightarrow P_{1} \rightarrow P_{0} \rightarrow G \rightarrow 0$ be a projective resolution of $G$. We form the double complex

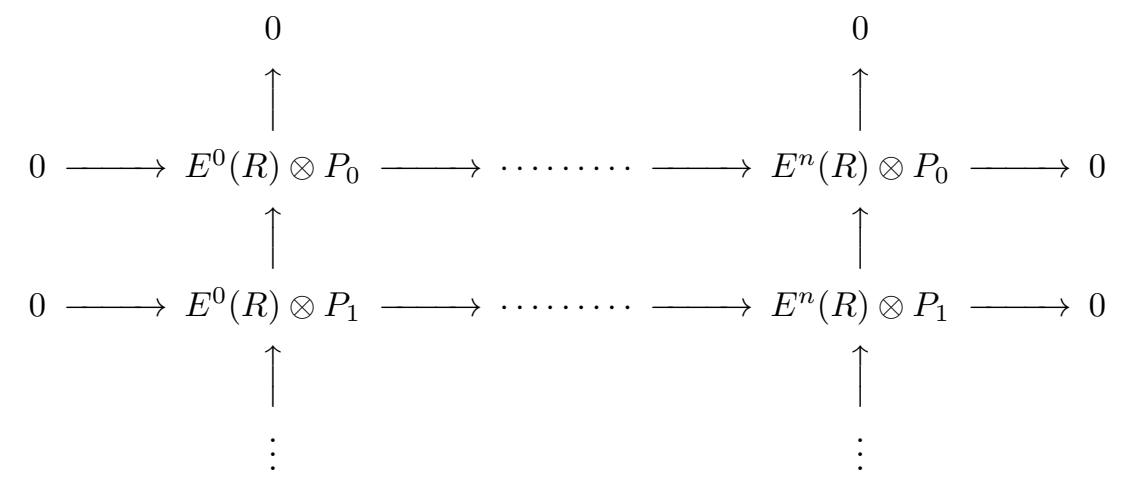

We now use a simple spectral sequence argument. First we note that this double complex can be regarded as a third quadrant double complex (using a shift in indices). This will guarantee convergence of our spectral sequences. For the $E^{1}$ term of our first spectral sequence we compute homology of this double complex by using the horizontal arrows. Since each $P_{n}$ is projective, and so flat, we now get the transpose of the diagram

$$
\cdots \rightarrow R \otimes P_{1} \rightarrow R \otimes P_{0} \rightarrow 0
$$

where all the missing terms are 0 . But now when we compute homology we just get $G$ (in the $(0,0)$ position).

We now first use the vertical arrows to compute homology. The terms we get will all be of the form $\operatorname{Tor}_{i}\left(E^{j}(R), G\right)$. By Proposition 2.2 and (3) these are 0 unless $i=$ $j$. Therefore, we get a diagonal double complex. Hence the horizontal differentials will be 0 , and when we compute homology again we get $\bigoplus_{k=0}^{n} \operatorname{Tor}_{k}\left(E^{k}(R), G\right)$. This means that $G$ has a filtration $0=G_{n+1} \subset G_{n} \subset \cdots \subset G_{1} \subset G_{0}=G$ with $G_{k} / G_{k+1} \cong \operatorname{Tor}_{k}\left(E^{k}(R), G\right)$ for $0 \leq k \leq n$. By Proposition 2.4 we know that each of these terms is Gorenstein injective.

By (3) $E^{k}(R)=\bigoplus_{P \in X_{k}} E(R / P)$ and so we have that $\operatorname{Tor}_{k}\left(E^{k}(R), G\right)$ $=\bigoplus_{P \in X_{k}} \operatorname{Tor}_{k}(E(R / P), G)$. Since each $E(R / P)$ has property $t(P)$ by $(5)$, so does $\operatorname{Tor}_{k}(E(R / P), G)$.

The uniqueness and functoriality will now follow from (7); i.e. if $P, Q$ are prime ideals of $R$ with $P \not \subset Q$, then $\operatorname{Hom}(S, T)=0$ whenever $S$ and $T$ have properties $t(P)$ and $t(Q)$ respectively.

We now indicate how this observation gives us the functoriality and uniqueness. Let $0 \subset G_{n} \subset \cdots G_{1} \subset G$ and $0 \subset H_{n} \subset \cdots \subset H_{1} \subset H$ be filtrations of the Gorenstein injective modules $G$ and $H$ satisfying the conclusion of the theorem. Let $S \subset G_{n}$ be the summand of $G_{n}$ corresponding to a given maximal ideal $P$ of $R$. Assume $n \geq 1$. Then we use the observation that $\operatorname{Hom}(S, U)=0$ if $U \subset H / H_{1}$ is the summand corresponding to some $Q \in X_{0}$. Since this holds for all such $U$ we get that $S \hookrightarrow G \rightarrow H / H_{1}$ is 0 . Therefore, $f(S) \subset H_{1}$. Since this is true for all the summands $S$ of $G_{n}$, we get that $f\left(G_{n}\right) \subset H_{1}$. But then we use this argument to get $f\left(G_{n}\right) \subset H_{2}, \ldots$ and finally that $f\left(G_{n}\right) \subset H_{n}$. 
Repeating the argument but applied to $G / G_{n} \rightarrow H / H_{n}$ with the induced filtration, we get that $f\left(G_{n-1}\right) \subset H_{n-1}$ and then by the induction hypothesis that $f\left(G_{k}\right) \subset H_{k}$ for $0 \leq k \leq n$.

Now if $P \in X_{k}$ and if $S$ and $T$ are the summands of $G_{k} / G_{k+1}$ and $H_{k} / H_{k+1}$ corresponding to $P$ respectively, then the same type of argument gives that $G_{k} / G_{k+1} \rightarrow$ $H_{k} / H_{k+1}$ maps $S$ into $T$.

The uniqueness of the filtrations and direct sum decompositions can be argued by assuming $G=H$ (with possibly different filtrations and direct sum decompositions) and letting $f=1_{G}$. So the above would give $G_{k} \subset H_{k}$. Then similarly we get $H_{k} \subset G_{k}$, and so $G_{k}=H_{k}$ for all $k$. Likewise we get the uniqueness of the direct sum decompositions.

Remark 3.2. We would like to thank the referee for help in writing this paper. The referee has pointed out that the $G_{k}$ of Theorem 3.1 can be described by the formulas $G_{k} / G_{k+1}=\bigoplus_{P \in X_{k}} \Gamma_{P}\left(G / G_{k+1}\right)$ for $k=0, \ldots, n$, where for a module $M$ we have that $\Gamma_{P}(M)$ consists of all $x \in M$ such that $P^{n} x=0$ for some $n \geq 1$. The referee also suggested that Theorem 3.1 might hold when we assume only the ring $R$ is Cohen-Macaulay, admitting a canonical module. We do not know if this is the case.

\section{Tensor Products of Gorenstein injective modules}

We let $R$ be a Gorenstein ring of finite Krull dimension $n$. We want to show that over such an $R$ all tensor products of Gorenstein injective modules are Gorenstein injective. If $G$ (or $H$ ) is a Gorenstein injective module and $0 \leq k \leq n+1$, then $G_{k}$ (or $H_{k}$ ) will denote the $k$-th submodule of $G$ (or $H$ ) that is part of the filtration provided by Theorem 3.1 .

Theorem 4.1. If $G$ and $H$ are Gorenstein injective modules, then so is $G \otimes H$.

Proof. If $S$ and $T$ are Gorenstein injective $R$-modules having properties $t(P)$ and $t(Q)$ respectively, then $S \otimes T=0$ if $P \neq Q$ (by (5)) and if $P=Q$ and $h t(P) \geq 1$ (by (9)). We use this to argue that $G \otimes H=G / G_{1} \otimes H / H_{1}$. This claim is trivial if $n=0$, since then $G_{1}=H_{1}=0$. So suppose $n \geq 1$. Then using the above and Theorem 3.1 we see that $G_{n} \otimes H_{k} / H_{k+1}=0$ for $k=0, \ldots, n$. Hence $G_{n} \otimes H=0$. Then tensoring the exact $0 \rightarrow G_{n} \rightarrow G \rightarrow G / G_{n} \rightarrow 0$ with $H$ we get that $G \otimes H=G / G_{n} \otimes H$.

If $n \geq 2$ (i.e. $n-1 \geq 1$ ), then the same argument gives that $G_{n-1} / G_{n} \otimes H=0$ and then that $G \otimes H=G / G_{n-1} \otimes H$. Continuing, we get that $G \otimes H=G / G_{1} \otimes H$. But then the same type argument gives that $G / G_{1} \otimes H=G / G_{1} \otimes H / H_{1}$ and so that $G \otimes H=G / G_{1} \otimes H / H_{1}$.

Now by Theorem 3.1 and (5) we see that $G \otimes H=G / G_{1} \otimes H / H_{1}$ will be a direct sum of modules of the form $S \otimes T$, where $S$ and $T$ both have property $t(P)$ for a minimal prime ideal $P$ of $R$. But such an $S$ and $T$ are naturally modules over $R_{P}$, and hence $S \otimes T$ is an $R_{P}$-module. Then by (8) $S \otimes T$ is a Gorenstein injective $R$-module. So finally noting that the class of Gorenstein injective modules is closed under direct sums (by (8)), we get that $G \otimes H$ is a Gorenstein injective $R$-module.

Remark 4.2. With the same hypothesis as in Theorem 4.1, we do not know if each $\operatorname{Tor}_{k}(G, H)$ is also Gorenstein injective when $k>0$. 


\section{ACKNOWLEDGEMEnts}

This paper was written while the second author was visiting the University of Kentucky from June to August, 2009. The second author was partially supported by the Specialized Research Fund for the Doctoral Program of Higher Education (Grant No. 20060284002), NSFC (Grant No. 10771095) and NSF of Jaingsu Province of China (Grant No. BK2005207).

\section{REFERENCES}

[1] H. Bass, On the ubiquity of Gorenstein rings, Math. Z. 82 (1963), 8-28. MR0153708 (27:3669)

[2] L.W. Christensen, Gorenstein Dimensions, Lecture Notes in Mathematics, 1747, SpringerVerlag, Berlin, 2000. MR 1799866 (2002e:13032)

[3] L.W. Christensen, H.-B. Foxby, and H. Holm, Beyond totally reflexive modules and back-a survey of Gorenstein dimensions, in "Commutative Algebra-Noetherian and non-Noetherian Perspectives", Springer-Verlag, New York, 2010, pp. 101-143.

[4] E.E. Enochs and O.M.G. Jenda, Gorenstein injective and projective modules, Math. Z. 220 (1995), 611-635. MR1363858 (97c:16011)

[5] E.E. Enochs and O.M.G. Jenda, Tensor and torsion products of injective modules, Journal of Pure and Applied Algebra 76 (1991), 143-149. MR.1145862 (93b:13013)

[6] E. Matlis, Injective modules over Noetherian rings, Pacific J. Math. 8 (1958), 511-528. MR0099360 (20:5800)

[7] J. Xu, Flat covers of modules, Lecture Notes in Mathematics, 1634, Springer-Verlag, Berlin, 1996. MR:1438789 (98b:16003)

Department of Mathematics, University of Kentucky, Lexington, Kentucky 40506

E-mail address: enochs@ms .uky .edu

Department of Mathematics, Nanjing University, Nanjing 210093, Jiangsu Province, People's Republic of China

E-mail address: huangzy@nju.edu.cn 